\title{
New Method of Measuring Coronary Diameter by Electron- Beam Computed Tomographic Angiography Using Adjusted Thresholds Determined by Calibration With Aortic Opacity
}

\author{
Nobusada Funabashi, MD; Yoshiki Kobayashi, MD*; Masayuki Kudo, RT**; \\ Miki Asano, MT; Kiyomi Teramoto, MD; Issei Komuro, MD; Geoffrey D Rubin, MD ${ }^{\dagger}$
}

\begin{abstract}
Background In a previous study the adjusted thresholds at which the diameters of coronary arteries determined by enhanced electron-beam computed tomography (CT) scans are equal to the corresponding quantitative coronary angiography measurements were analyzed, and their correlation with maximum CT values for the vessel short axes was determined. A rapid accurate method for such measurements was sought by substituting maximum CT values for the descending aorta in the corresponding axial images for those for the short axes.

Methods and Results In 8 patients, 179 sites were measured. Means $( \pm$ SD) of adjusted thresholds and the maximum CT values for vessel short axes and the descending aorta in the corresponding axial images for all vessels were 108 $\pm 66,227 \pm 80$, and $363 \pm 75$ Hounsfield Unit (HU), respectively. Adjusted thresholds correlated with the maximum CT values for the corresponding vessel short axes and the descending aorta in the corresponding axial images, with $\mathrm{R}^{2}=0.55,0.33, \mathrm{p}<0.01$, respectively. An abbreviated formula for use of maximum $\mathrm{CT}$ values for the descending aorta in the corresponding axial images was $\mathrm{y}=0.5 \mathrm{x}-75$ (HU) ( $\mathrm{y}=$ adjusted threshold, $\mathrm{x}=$ maximum CT value for the descending aorta in the corresponding axial image).
\end{abstract}

Conclusions The abbreviated formula provided a rapid, accurate method for measurements independent of arterial enhancement. (Circ J 2004; 68: 769-777)

Key Words: Adjusted thresholds; Coronary artery; Electron beam computed tomography; Quantitative coronary angiography

M easurements of the diameters of 3-dimensional (3-D) coronary arteries using electron-beam computed tomography (EBCT) and shaded surface display (SSD) techniques have been qualitatively compared with those obtained by cine coronary angiography!-3 The threshold selection has been set at $80-100$ Hounsfield Units (HU) and from quantitative analysis using different threshold selections, ${ }^{4}$ it is reasonable to expect that the optimal threshold level for SSD depends on the magnitude of arterial enhancement. As a result, we hypothesized that a single threshold setting would not constitute a reliable measurement of coronary arterial luminal diameter using EBCT. We have previously reported that adjusted threshold methods using line density profile (LDP) curves in consideration of arterial enhancement provided more accurate results than fixed threshold methods 5,6 However, in routine practice it is unrealistic to make an LDP curve, or to measure the maximum computed tomo-

(Received February 24, 2004; revised manuscript received May 13, 2004; accepted May 28, 2004)

Department of Cardiovascular Science and Medicine, Chiba University Graduate School of Medicine, Chiba, *First Department of Internal Medicine, Osaka City University, Osaka, ${ }^{* * G E}$ Yokokawa Medical Systems, Tokyo and Department of Radiology, Stanford University School of Medicine, California, USA

Mailing address: Nobusada Funabashi, MD, Department of Cardiovascular Science and Medicine, Chiba University Graduate School of Medicine, 1-8-1 Inohana, Chuo-ku, Chiba, Chiba 260-8670, Japan.

E-mail: nobusada@ma.kcom.ne.jp graphy (CT) value for the short axis at each site in the coronary arteries.

In the present study, we analyzed the adjusted thresholds at which the diameters of coronary arteries determined by EBCT scans are equal to the corresponding quantitative coronary angiography (QCA) measurements. Calibration using aortic opacity of the opacified blood, and using maximum CT values for the descending aorta in the corresponding axial images as a substitute for the maximum CT values for the short axes of the coronary arteries, was performed to accurately resolve the measurements of coronary artery luminal diameter. Furthermore we sought to establish a simplified method for measuring coronary artery diameters that was independent of inter- and intrapatient variations in arterial enhancement.

\section{Methods}

\section{Patients}

Under a protocol approved by the institutional committee for the protection of human subjects and after informed consent was obtained, 8 male patients aged 53-63 years old, who had previously undergone heart transplantation at a mean 3.6 years prior to enrollment, were recruited to undergo both EBCT (Imatron C-150XP, Imatron, South San Francisco, CA, USA) and cine coronary angiography within a 24-h period. 

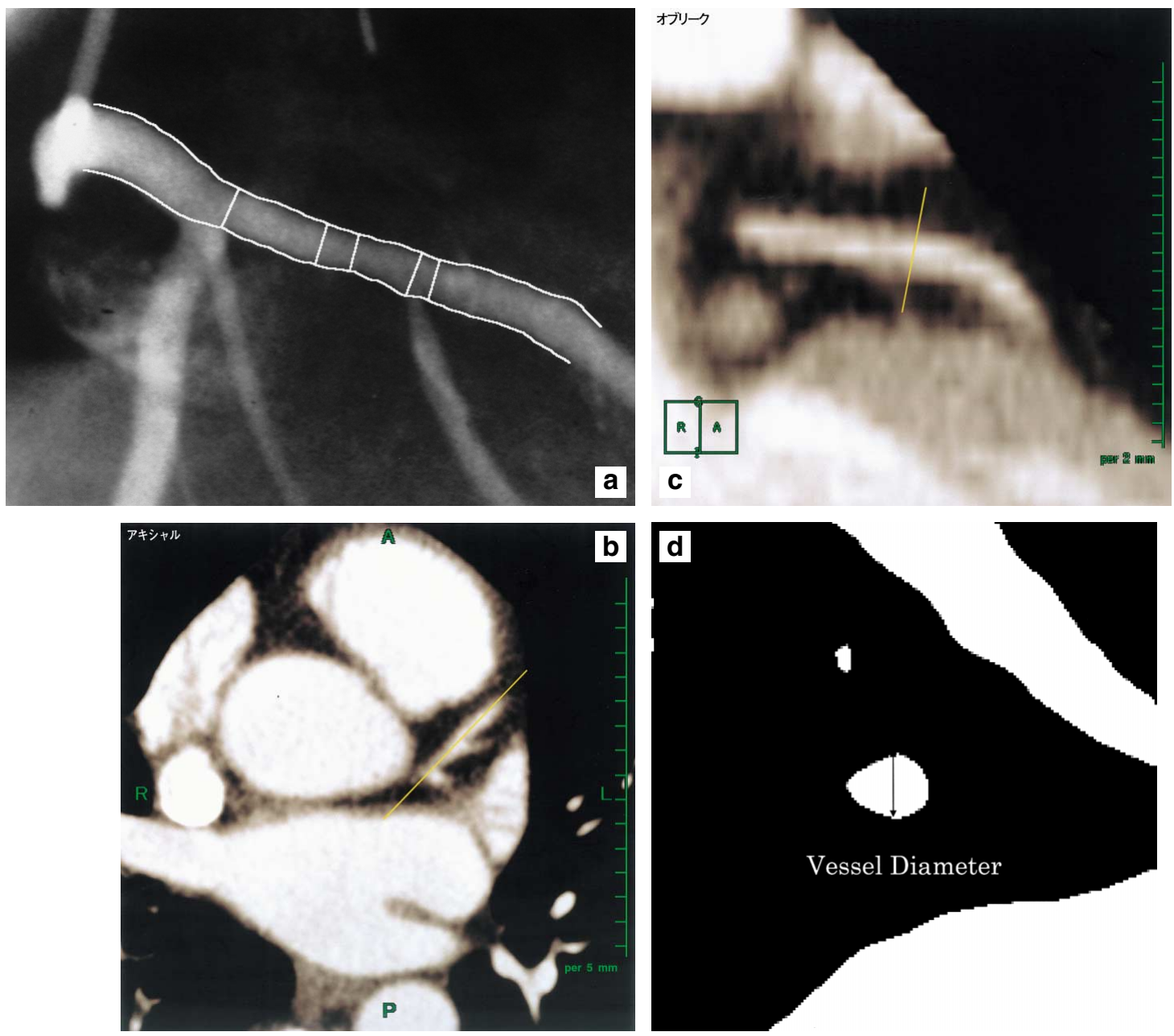

Fig 1. (a) Quantitative coronary angiogram showing the 30-degree right anterior oblique projection of the left coronary arteries. Lines indicate manual tracing. (b-d) Schema of measurement of adjusted thresholds using electron-beam computed tomography (CT). (b) Transverse source image obtained with contrast-enhanced electron-beam CT. The yellow line indicates the long axis of the LAD branch with which we measured vessel diameters. (c) Oblique planar image parallel to the long axis of the LAD branch. The yellow line indicates the axis perpendicular to the long axis of the vessel. Diameters were measured perpendicular to the median centerline of the vessel. Oblique planar reformations were generated parallel to the long axis of vessel, which approximates the oblique projections analyzed with cine coronary angiography. (d) Oblique planar image perpendicular to the long axis of the LAD branch. By setting the display width at zero, we were able to reduce the grayscale to black and white. We set the window levels at the site at which the vessel diameters were equal to the diameters measured by quantitative coronary angiography (QCA) measurement; these window levels were regarded as the adjusted thresholds. Vessel diameters were measured at each measurement site in the same direction as for the coronary angiograms.

\section{Cine Coronary Angiography}

Cine coronary angiography was performed using the percutaneous femoral approach. Sublingual nitroglycerin $(0.4 \mathrm{mg})$ was given $2-3 \mathrm{~min}$ before the contrast injection, to minimize the effect of varying vasomotor tone on vessel lumen diameters. Catheters of known diameters were used for calibration. Multiple projections, including cranial and caudal angulated views, were obtained for all patients. The 30-degree right anterior oblique (RAO) projection was used to assess the left coronary artery (LCA) and its branches, and the 60-degree left anterior oblique (LAO) projection was used to assess the right coronary artery (RCA).

QCA was performed off-line using a computerized edge detection program (QCA plus, Sanders Data System, Palo Alto, CA, USA) developed and validated at Stanford ${ }^{7}$ and analyzed by a cardiologist (Y.K.) unaware of the EBCT results (Fig 1a). Diameters of the proximal left main (LM) artery and its distal end just above the bifurcation of the left anterior descending branch (LAD) and the left circumflex branch (LCX) were recorded, as well as the diameter at a point between these 2 sites. Also measured were the diameters of the LAD, the LCX, and the RCA, each at their proximal ends and at 2-4 equally spaced points between their proximal ends and the first atrial side branch. Single end-diastolic cine frames, identified by an electrocardiogram-triggered mark on the frames and selected for optimal coronary vessel opacification, were focused and magnified. The digitized image was displayed on a graphic computer terminal linked to a light pen, which was used to manually trace the margins of either the catheter or coronary segment. Using these lines as initial search locations, the automatic edge-finding algorithm drew and smoothed the edges, defining the edge as the peak of the first derivative of the gray-scale density gradient, perpendicular to the long axis 


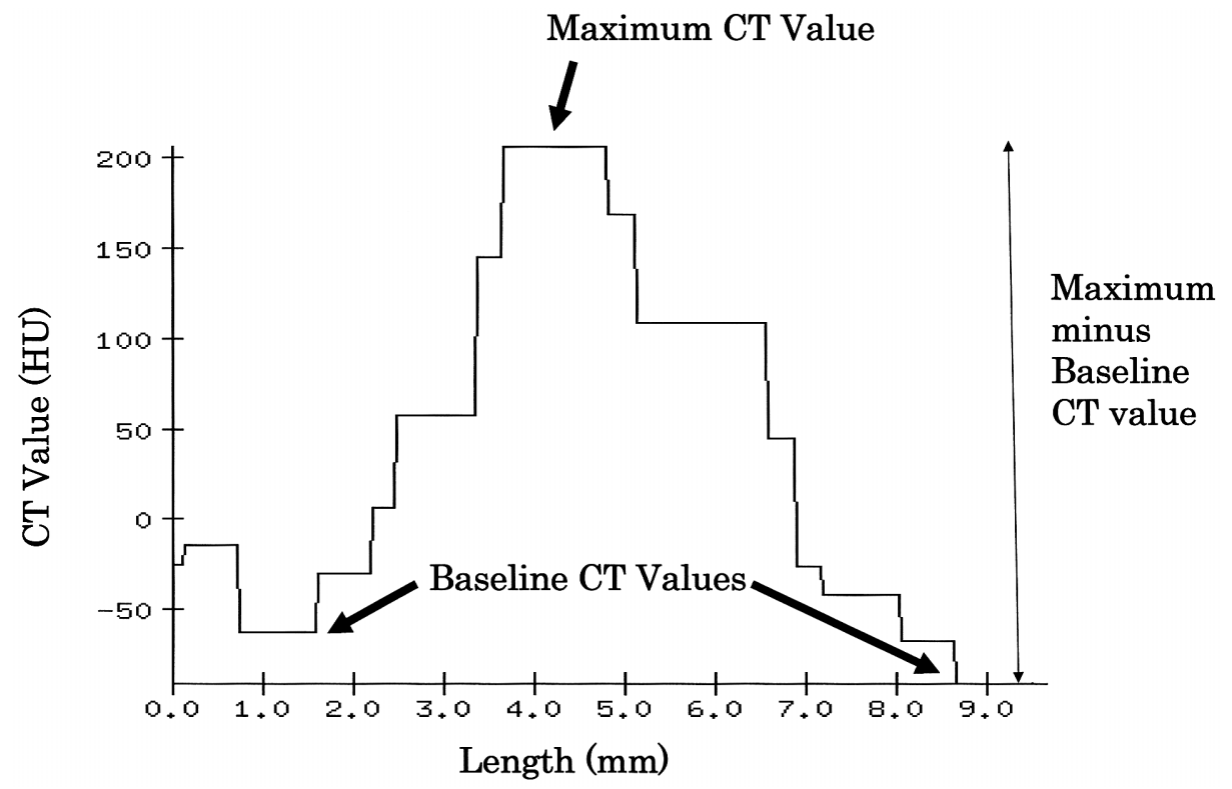

Fig 2. Schema of line density profile (LDP) method. Plot shows the voxel values along a line that is perpendicular to the median centerline of the vessel at each measurement site. In this schema, the baselines of the LDP were established as the epicardial fat immediately adjacent to the vessel on both sides. For the LDP curve, baseline computed tomography (CT) values were calculated for the right side and the left side in addition to the maximum CT value represented as the schema. We measured the maximum CT value minus baseline CT value at both sides and used both the larger and average values.

of the catheter or vessel, as estimated from the initial manual tracings. When the computer algorithm was unable to resolve vessel boundaries in areas of noise or vessel crossings, manual editing of short segments of boundary with the light pen was used to correct the computer-generated boundary. At no time did the length of a manually entered margin exceed $20 \%$ of the total length of the quantitated segment. After the light pen indicated the start and end of the segment, the mean diameter of the segment was computed from perpendiculars constructed through the length of a computer-generated centerline. The distance of each measurement point from the LM or the RCA ostium was measured.

\section{EBCT Measurement}

EBCT attains ultrafast scan speeds by sweeping a steered electron beam on a fixed tungsten target ring, thereby providing a moving $\mathrm{X}$-ray source without mechanical motion? The 100-ms modes are used for high-resolution cross-section imaging in a Step-Volume Scan mode with electrocardiogram gating? EBCT was coupled to a $160 \mathrm{ml}$ intravenous injection of iodinated contrast material $(300 \mathrm{mg} / \mathrm{ml})$ performed during breath holding, and was directed over $60-70 \mathrm{~mm}$ of the proximal and middle portions of the coronary arteries using $1.5 \mathrm{~mm}$ collimation, $1.0 \mathrm{~mm}$ table incrementation, and triggered to $80 \%$ of the $\mathrm{R}$-to- $\mathrm{R}$ interval. The patients received nitroglycerin prior to EBCT, as in the cine angiography studies.

EBCT data were transferred to a workstation (AdvantageWindows, General Electronics, Milwaukee, WI, USA) and 18-27 measurement points per patient within the coronary tree were defined relative to identifiable branches.

Vessel diameters were measured at each measurement site in the same direction as the QCA measurements. Diameters were analyzed by a cardiologist (N.F.) unaware of the QCA results, using the workstation program described later (Fig 1 b-d). Double oblique planar reformations were generated, firstly parallel to the long axes of the vessels, approximating the oblique projections analyzed by cine coronary angiography $(\mathrm{Fig} 1 \mathrm{~b}, \mathrm{c})$. Using the vessel origins and the major branch sites as landmarks, the loci corresponding to the QCA measurement were identified on the EBCT scans. Second, an additional oblique planar reformation was generated perpendicular to the first oblique reformation image (long axes of the vessel) (Fig 1c). By setting the display width at zero, we were able to reduce the grayscale to black and white (Fig 1d). We set the window levels at the site at which vessel diameters were equal to the diameters obtained by QCA measurement; these window levels were regarded as the adjusted thresholds. Furthermore, LDP curves were made perpendicular to the median centerline of the vessel at each measurement site. A plot of the voxel values along a line perpendicular to the vessel at each measurement site was created (Fig 2). The baseline of the LDP was established as epicardial fat or myocardium immediately adjacent to the artery. For the LDP curve, baseline CT values were calculated for the right and left sides in addition to the maximum $\mathrm{CT}$ value. We measured the maximum CT value minus baseline CT value using the larger and average values for both sides in the scatter plot. The maximum CT value for the short axis of the coronary arterial lumen at each measurement site was measured from the oblique planar reformations perpendicular to the long axis of the vessel. Also, the maximum CT value for the descending aorta at the corresponding axial level of each measurement site was measured. As some calcifications, which apparently caused a higher attenuation than the vessel lumen, were evident on the enhanced axial images of the descending aorta, we carefully excluded them and measured the maximum CT values for the descending aorta. The maximum CT value was used as an index for assessing arterial enhancement. 
Table 1 QCA Diameter With Distance From the Ostium

\begin{tabular}{lccc}
\hline \hline Vessel type & $n$ & QCA Diameter $(\mathrm{mm})$ & Distance from Ostium $(\mathrm{mm})$ \\
\hline LM & 11 & $5.07 \pm 0.75(3.82-6.09)$ & $3.21 \pm 2.9(0-9.39)$ \\
LAD & 62 & $3.3 \pm 0.85(1.46-5.28)$ & $34.64 \pm 17.13(5-77.64)$ \\
LCX & 46 & $3.53 \pm 0.76(1.52-5.27)$ & $23.81 \pm 15.16(3-62.54)$ \\
Total & 60 & $3.61 \pm 0.58(2.71-5.67)$ & $28.56 \pm 20.93(0-79.77)$ \\
& 179 & $3.57 \pm 0.84(1.46-6.09)$ & $27.89 \pm 19.04(0-79.77)$ \\
\hline
\end{tabular}

QCA, quantitative coronary arterigraphy; LM, left main; LAD, left anterior descending branch; LCX, left circumflex branch; $R C A$, right coronary artery.

Table 2 QCA Diameter, Distance From the Ositum, Adjusted Threshold, and Maximum CT Values for Each Patient

\begin{tabular}{|c|c|c|c|c|c|c|}
\hline $\begin{array}{l}\text { Patient } \\
\text { no. }\end{array}$ & $n$ & $\begin{array}{l}\text { QCA Diameter } \\
(\mathrm{mm})\end{array}$ & $\begin{array}{l}\text { Distance from } \\
\text { ostium (mm) }\end{array}$ & $\begin{array}{l}\text { Adjusted threshold } \\
(H U)\end{array}$ & $\begin{array}{l}\text { Maximum CT value } \\
\text { in short axis }(H U)\end{array}$ & $\begin{array}{c}\text { Maximum } C T \text { value } \\
\text { in aorta }(H U)\end{array}$ \\
\hline 1 & 18 & $3.31 \pm 1.19$ & $22.2 \pm 16.3$ & $138 \pm 44$ & $214 \pm 37$ & $344 \pm 28$ \\
\hline 2 & 23 & $3.25 \pm 0.73$ & $25.9 \pm 17.4$ & $80 \pm 35$ & $169 \pm 57$ & $293 \pm 22$ \\
\hline 3 & 20 & $4.06 \pm 0.9$ & $20.1 \pm 17.6$ & $148 \pm 32$ & $282 \pm 33$ & $376 \pm 31$ \\
\hline 4 & 20 & $3.57 \pm 0.46$ & $25.2 \pm 16.6$ & $85 \pm 46$ & $210 \pm 37$ & $374 \pm 14$ \\
\hline 5 & 26 & $3.57 \pm 0.69$ & $30.2 \pm 16.9$ & $196 \pm 41$ & $327 \pm 92$ & $475 \pm 40$ \\
\hline 6 & 24 & $3.57 \pm 0.56$ & $32.5 \pm 21.4$ & $69 \pm 39$ & $243 \pm 65$ & $412 \pm 25$ \\
\hline 7 & 21 & $3.57 \pm 0.48$ & $26.1 \pm 15.7$ & $110 \pm 59$ & $213 \pm 56$ & $349 \pm 27$ \\
\hline 8 & 27 & $3.57 \pm 0.64$ & $36.2 \pm 21.8$ & $48 \pm 59$ & $155 \pm 43$ & $276 \pm 76$ \\
\hline Total & 179 & $3.57 \pm 0.84$ & $27.9 \pm 19$ & $108 \pm 66$ & $227 \pm 80$ & $363 \pm 75$ \\
\hline
\end{tabular}

QCA, quantutative coronary arteriography; HU, Hounsfield Units.

Table 3 QCA Diameter, Distance From the Ostium, Adjusted Threshold, and Maximum CT Values for Each Vessel

\begin{tabular}{lcccccc}
\hline \hline $\begin{array}{l}\text { Vessel } \\
\text { site }\end{array}$ & $n$ & $\begin{array}{c}\text { QCA diameter } \\
(\mathrm{mm})\end{array}$ & $\begin{array}{c}\text { Distance from } \\
\text { ostium }(\mathrm{mm})\end{array}$ & $\begin{array}{c}\text { Adjusted threshold } \\
(\mathrm{HU})\end{array}$ & $\begin{array}{c}\text { Maximum CT value } \\
\text { in short axis }(\text { HU })\end{array}$ & $\begin{array}{c}\text { Maximum CT value } \\
\text { in aorta }(\mathrm{HU})\end{array}$ \\
\hline LM & 11 & $5.07 \pm 0.72$ & $3.2 \pm 2.8$ & $112 \pm 42$ & $263 \pm 71$ & $349 \pm 49$ \\
$L A D$ & 62 & $3.3 \pm 0.84$ & $34.6 \pm 17$ & $107 \pm 69$ & $207 \pm 71$ & $338 \pm 82$ \\
$\quad<30$ mm from ostium & 25 & $3.88 \pm 0.74$ & $18.7 \pm 7.4$ & $79 \pm 64$ & $204 \pm 71$ & 33778 \\
$\quad \geq 30$ mm from ostium & 37 & $2.92 \pm 0.67$ & $45.4 \pm 12.6$ & $127 \pm 65$ & $209 \pm 72$ & $339 \pm 86$ \\
LCX & 46 & $3.53 \pm 0.75$ & $23.8 \pm 15$ & $85 \pm 55$ & $203 \pm 52$ & $368 \pm 63$ \\
$\quad<30$ mm from ostium & 30 & $3.66 \pm 0.74$ & $14.3 \pm 6.5$ & $80 \pm 49$ & $208 \pm 46$ & $355 \pm 62$ \\
$\quad 30$ mm from ostium & 16 & $3.29 \pm 0.72$ & $41.6 \pm 9$ & $94 \pm 63$ & $192 \pm 61$ & $393 \pm 58$ \\
$R C A$ & 60 & $3.61 \pm 0.57$ & $28.6 \pm 20.8$ & $126 \pm 70$ & $259 \pm 93$ & $387 \pm 69$ \\
$\quad<30$ mm from ostium & 31 & $3.81 \pm 0.61$ & $11.6 \pm 9.3$ & $118 \pm 78$ & $256 \pm 93$ & $371 \pm 67$ \\
$\quad 30$ mm from ostium & 29 & $3.4 \pm 0.44$ & $46.7 \pm 12.7$ & $135 \pm 59$ & $262 \pm 92$ & $404 \pm 68$ \\
Total & 179 & $3.57 \pm 0.84$ & $27.9 \pm 19$ & $108 \pm 66$ & $227 \pm 80$ & $363 \pm 75$
\end{tabular}

QCA, quantitative coronary arteriography; LM, left main; LAD, left anterior descending branch; LCX, left circumflex branch; RCA, right coronary artery; HU, Hounsfield Units.

\section{Statistical Analysis}

Adjusted thresholds were correlated with arterial enhancement (maximum CT values for the vessel short axes, the descending aorta in the corresponding axial images, vessel sizes (the corresponding QCA diameter measurements), and the distances from the vessel ostium. Variations between the 2 groups (adjusted thresholds and thresholds according to the abbreviated formula) were compared using the $\mathrm{F}$ distribution test.

\section{Results}

\section{QCA Diameters With Distances From the Ostium}

All 8 cine coronary angiograms were interpreted as being normal. A total of 179 sites were measured among the 8 patients (LM, 11; LAD, 62; LCX, 46; RCA, 60).

Table 1 lists the QCA diameters with the distances from the LM or RCA ostium. The QCA diameters ranged from $1.46 \mathrm{~mm}$ to $6.09 \mathrm{~mm}$ (mean $\pm \mathrm{SD}, 3.57 \pm 0.84 \mathrm{~mm})$. Distances from the ostium ranged from 0 to $79.77 \mathrm{~mm}(27.9 \pm$ $19.04 \mathrm{~mm})$.

\section{EBCT Measurement With Arterial Enhancement}

Table 2 lists the number of vessel sites, QCA diameters (mean $\pm \mathrm{SD}$ ), distances from the ostium, adjusted thresholds, and maximum CT values for the vessel short axes and the descending aorta in the corresponding axial images for each patient.

Table 3 lists the number of vessel sites, distances from the ostium (mean $\pm \mathrm{SD}$ ), adjusted thresholds and maximum CT values for the vessel short axes and the descending aorta in the corresponding axial images for each vessel type. Values were further stratified according to the position of measurement from the vessel ostium. Adjusted thresholds, maximum CT values for vessel short axes and maximum CT values for the descending aorta in the corresponding axial images for all vessels were $108 \pm 66,227 \pm$ 80 , and $363 \pm 75 \mathrm{HU}$, respectively.

All sites corresponding to the maximum $\mathrm{CT}$ values for both the vessel short axes and the descending aorta in the corresponding axial images were located approximately at the center of the vessel lumen. After review of the cine coronary angiograms and the enhanced axial images of the 

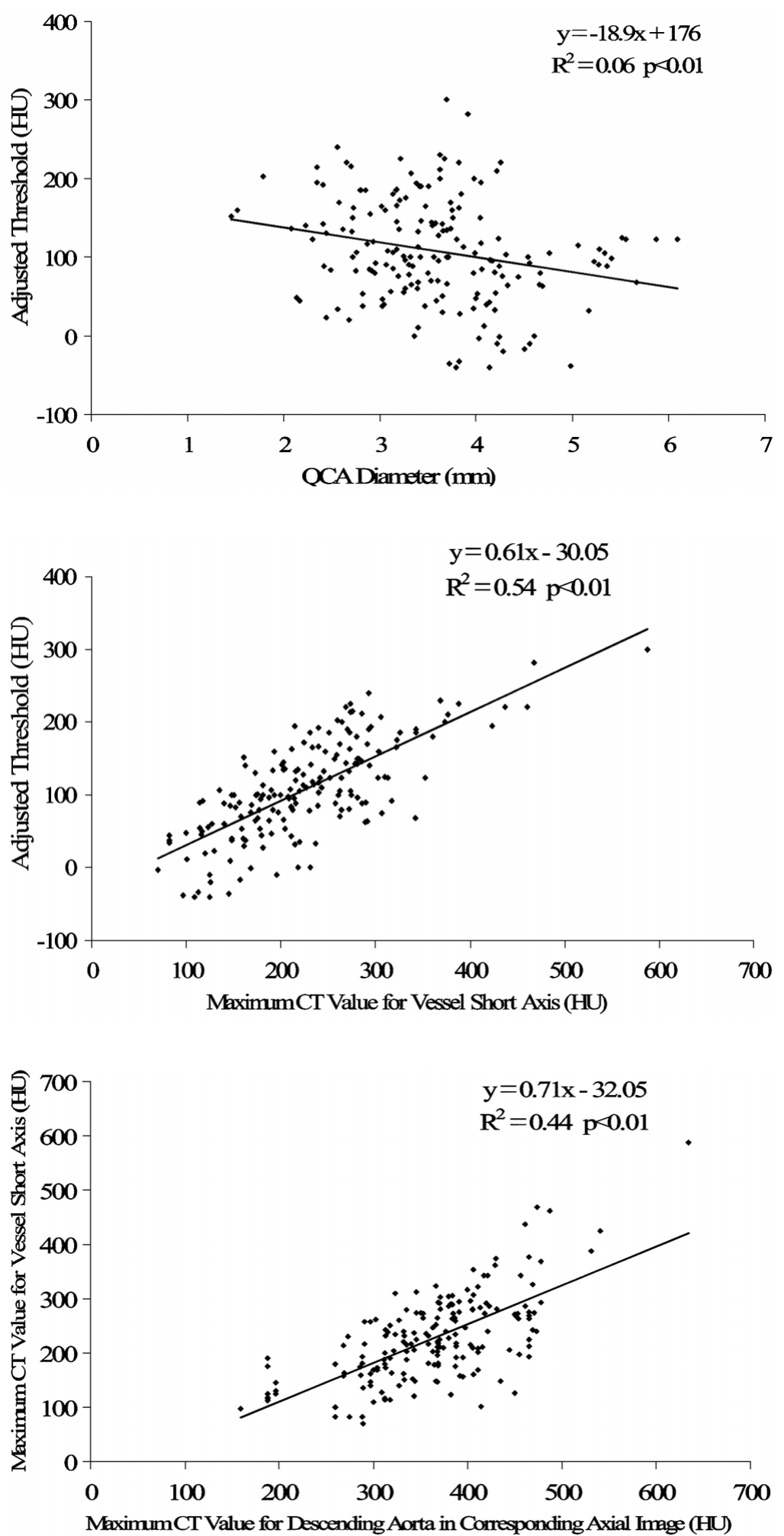

Fig 3. Scatter plot of adjusted threshold vs quantitative coronary angiography (QCA) diameter. The adjusted thresholds very weakly correlated with the QCA diameters $\left(\mathrm{R}^{2}=0.06, \mathrm{p}<0.01\right)$. These mean values were distributed about a straight line given by $\mathrm{y}=18.9 \mathrm{x}-176$.

Fig 4. Scatter plot of adjusted threshold vs maximum computed tomography (CT) value for the corresponding vessel short axis. The adjusted thresholds correlated with the maximum $\mathrm{CT}$ values for the corresponding vessel short axes $\left(\mathrm{R}^{2}=0.54, \mathrm{p}<0.01\right)$.

Fig 5. Scatter plot of maximum computed tomography $(\mathrm{CT})$ value for the vessel short axis with maximum CT value for the descending aorta in the corresponding axial image. The maximum CT values for the vessel short axes correlated with those for the descending aorta in the corresponding axial images $\left(\mathrm{R}^{2}=0.44, \mathrm{p}<0.01\right)$. These mean values were distributed about a straight line given by $\mathrm{y}=0.71 \mathrm{x}-32.1$.

EBCT data sets, we concluded that the possibility of the presence of calcification or artifact in the coronary arteries might be low; nevertheless, we were careful not to adopt the CT values relating to calcification when calculating maximum $\mathrm{CT}$ values for the descending aorta.

As shown in Fig 3, the adjusted thresholds correlated very weakly with QCA diameters, with $\mathrm{R}^{2}=0.06, \mathrm{p}<0.01$. These mean values were distributed about a straight line given by $\mathrm{y}=-18.9 \mathrm{x}+176$.

The adjusted thresholds correlated with maximum CT values for the corresponding vessel short axes, with $\mathrm{R}^{2}=$ $0.54, \mathrm{p}<0.01$ (Fig 4). These mean values were distributed about a straight line given by $y=0.61 x-30.1$. As shown in Fig 5, the maximum CT values for the vessel short axes correlated with those for the descending aorta in the corresponding axial images with $\mathrm{R}^{2}=0.44, \mathrm{p}<0.01$. These mean 

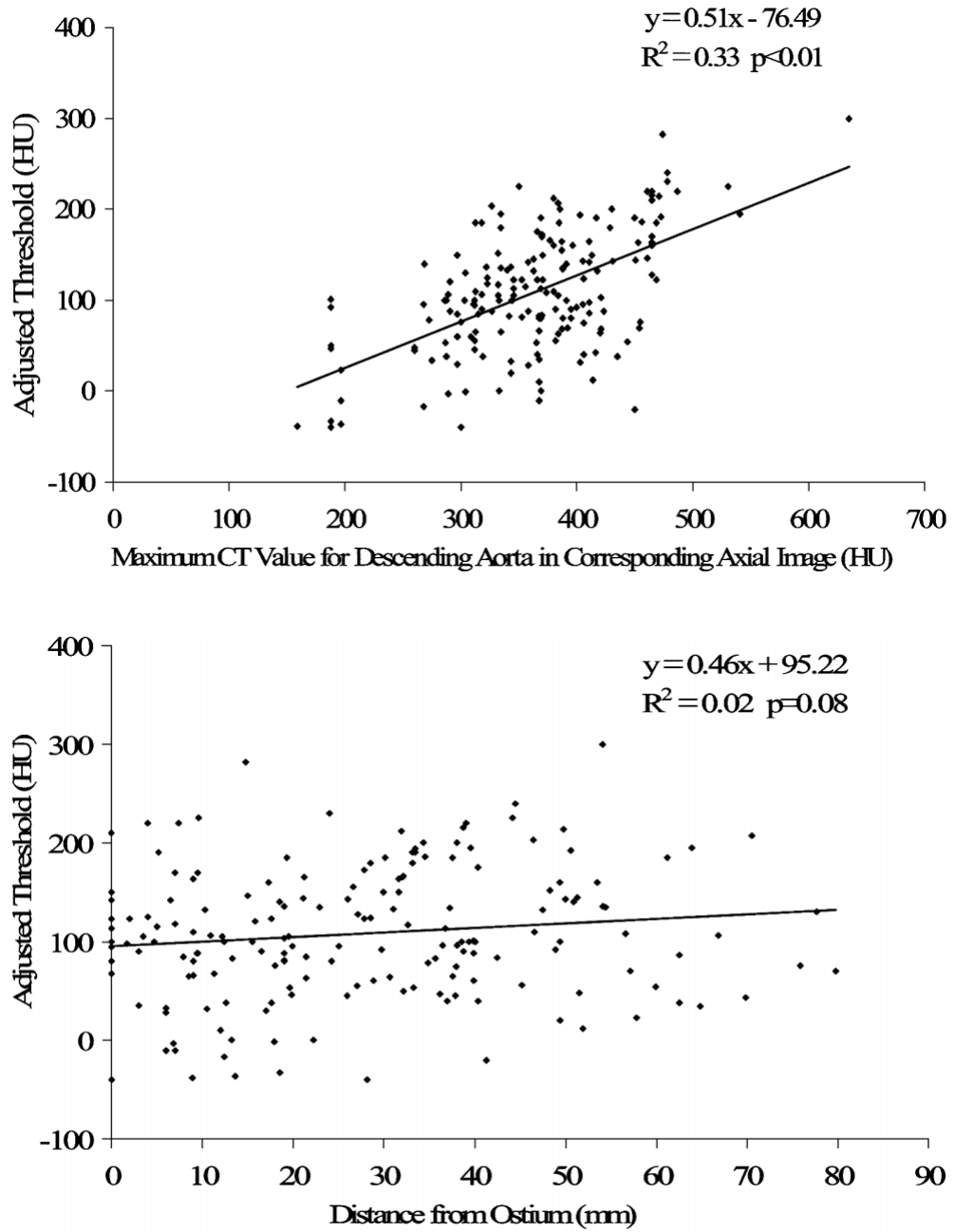

Fig 6. Scatter plot of adjusted threshold with maximum computed tomography (CT) value for the descending aorta in the corresponding axial image. The adjusted thresholds correlated with the maximum CT values for the descending aorta in the corresponding axial images $\left(\mathrm{R}^{2}=0.33, \mathrm{p}<0.01\right)$. These mean values were distributed about a straight line given by $y=0.51 x-76.5$. The slope of the adjusted thresholds vs the maximum CT values for the descending aorta in the corresponding axial images was approximately 0.5 . The intercept of this line was $-76.5 \mathrm{HU}$.
Fig 7. Scatter plot of adjusted threshold vs distance from the ostium. The adjusted thresholds did not correlate significantly with distances from the ostium $\left(\mathrm{R}^{2}=0.02, \mathrm{p}=0.08\right)$. values were distributed about a straight line given by $\mathrm{y}=0.71 \mathrm{x}-32.1$.

The adjusted thresholds correlated with the maximum CT values for the descending aorta in the corresponding axial images with $\mathrm{R}^{2}=0.33$, $\mathrm{p}<0.01$ (Fig 6). These mean values were distributed about a straight line given by $y=0.51 x-76.5$. The slope of the adjusted thresholds vs the maximum CT values for the descending aorta in the corresponding axial images was approximately 0.5 . The intercept of this line was $-76.5 \mathrm{HU}$.

As shown in Fig7, the thresholds did not correlate significantly with distances from the ostium, with $\mathrm{R}^{2}=0.02$, $\mathrm{p}=0.08$.

As shown in Fig 8a, the adjusted thresholds correlated with the maximum minus the baseline $\mathrm{CT}$ values in the LDP (average of the 2 sides), with $\mathrm{R}^{2}=0.30, \mathrm{p}<0.01$. These mean values were distributed about a straight line given by $y=0.43 x-15.8$. As shown in Fig $8 b$, the adjusted thresholds correlated with the maximum minus the baseline CT values in the LDP (larger of the 2 sides), with $\mathrm{R}^{2}=0.37, \mathrm{p}<0.01$. These mean values were distributed about a straight line given by $\mathrm{y}=0.47 \mathrm{x}-40.4$.

From the straight line given by $\mathrm{y}=0.51 \mathrm{x}-76.5$ in Fig 6 , ( $y=$ adjusted threshold, $\mathrm{x}=$ maximum $\mathrm{CT}$ value for the descending aorta in the corresponding axial images), we determined the thresholds according to the abbreviated formula whereby $y=0.5 x-75$ (HU) $(y=$ adjusted threshold, $\mathrm{x}=$ maximum $\mathrm{CT}$ value for the descending aorta in the corresponding axial image). The constant, $-75 \mathrm{HU}$, may reflect a complex of various baseline (or background) factors such as epicardial fat or myocardium. The mean $( \pm \mathrm{SD})$ baseline CT values of the LDP of the total, the group considered to be epicardial fat and the group considered to be myocardium, were $-61.0 \pm 60.4,-82.5 \pm 38.6$, and $41.6 \pm 35.3 \mathrm{HU}$ with ranges of -181 to $150,-181$ to -2 , and 0 to $150 \mathrm{HU}$, respectively.

As shown in Fig 9, the adjusted thresholds correlated with the thresholds determined by the abbreviated formula, with $\mathrm{R}^{2}=0.33 \mathrm{p}<0.01$. These mean values were distributed about a straight line given by $y=1.02 x-0.4$. The slope of the adjusted thresholds vs the thresholds according to the abbreviated formula was approximately 1.0, and the intercept of this line was only $-0.4 \mathrm{HU}$. In addition, the mean \pm SD of thresholds by the abbreviated formula was $107 \pm$ $37 \mathrm{HU}$. In comparing the mean \pm SD of the adjusted thresholds, the mean of the thresholds by the abbreviated formula was similar (107 vs $108 \mathrm{HU})$, but the standard deviation was significantly smaller ( 37 vs $66 \mathrm{HU})$.

As shown in Fig 10, the CT angiography diameter correlated with QCA diameter $\left(\mathrm{R}^{2}=0.09 \mathrm{p}<0.01\right)$. The mean values were distributed about a straight line given by $\mathrm{y}=0.50 \mathrm{x}+1.86$.

\section{Discussion}

Cine coronary angiography has been the standard for 


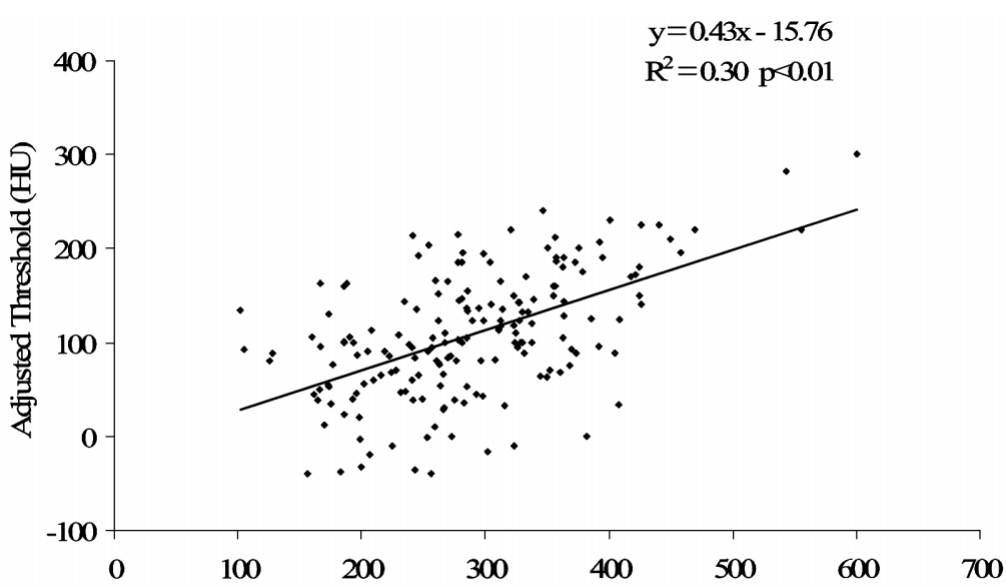

Maximumminus Baseline CT Value of LDP (Average of the Two Sides) (HU)
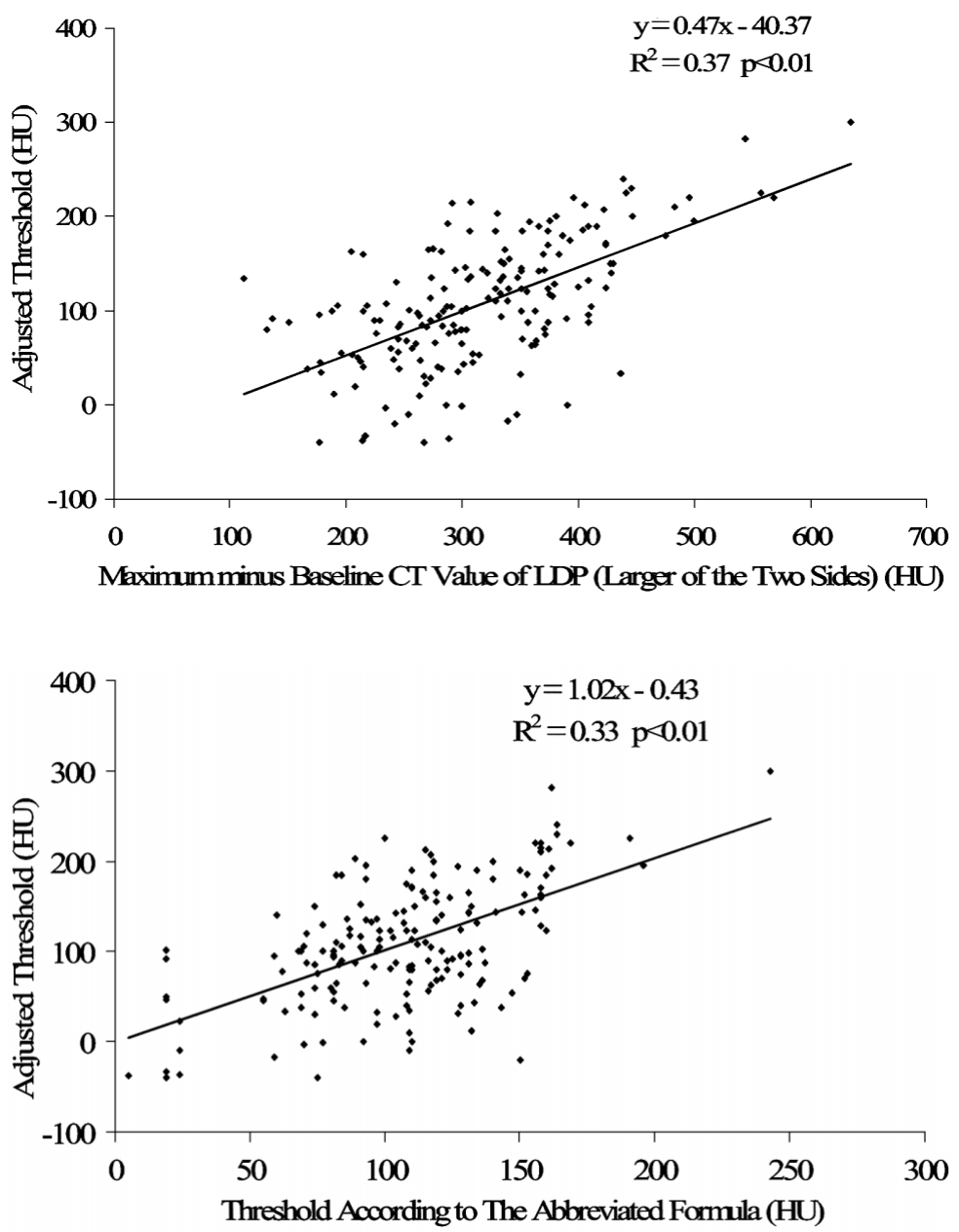

Fig 8. Scatter plots of adjusted threshold vs maximum minus the baseline computed tomography $(\mathrm{CT})$ value in the line density profile (LDP) ((a) average of the 2 sides) and ((b) larger of the 2 sides). In (a) and (b), the adjusted thresholds were correlated with the maximum minus baseline CT values in the LDP $\left(\mathrm{R}^{2}=0.30, \mathrm{p}<0.01\right.$ and $\mathrm{R}^{2}=0.37, \mathrm{p}<0.01$, respectively). LDP indicates line density profile.

Fig 9. Scatter plot of adjusted threshold vs threshold obtained by an abbreviated formula. The adjusted thresholds correlated with threshold according to the formula $\left(\mathrm{R}^{2}=0.33, \mathrm{p}<0.01\right)$. The mean values were distributed about a straight line given by $y=1.02 x-0.4$. The slope of the line was approximately 1.0 and the intercept was $-0.4 \mathrm{HU}$. evaluating the extent of coronary artery disease, but recently 3 -D visualization of coronary arteries using EBCT has emerged as a potential non-invasive alternative!,2,10 Although qualitative reports have been published regarding the utility of EBCT coronary angiography,1-13 there are few reports of quantitative analysis of EBCT coronary angiography as compared with cine coronary angiography,6 We have speculated that several factors may influence the absolute luminal caliber of coronary arteries on EBCT angiograms, such as arterial enhancement, vessel diameter, distance from the ostium, and vessel types triggered to contract at the end of diastole.

Because the acquisition time requires $40-80$ heartbeats, there must be significant differences in the state of the enhancement at the level of each slice ${ }^{14}$ We first made a short-axis image of the coronary arteries at each site and measured the adjusted threshold at which vessel diameter was equal to that obtained from the corresponding QCA measurement.

Even though the total mean of the adjusted threshold was $108 \mathrm{HU}$, we concluded that a single threshold setting could not be used reliably to measure the luminal diameter 


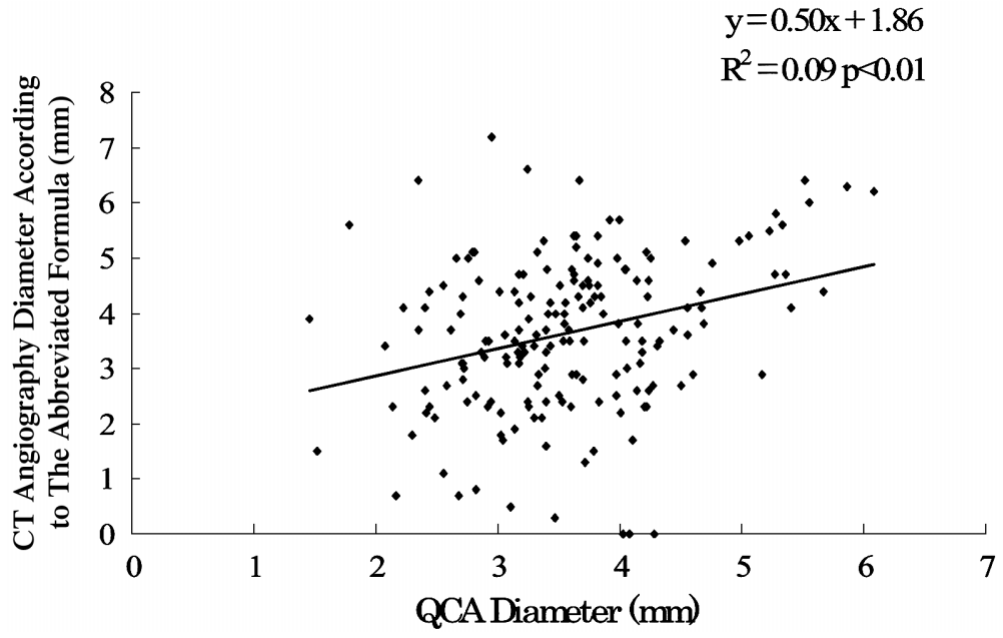

Fig 10. Scatter plot of computed tomography (CT) angiography diameter according to the abbreviated formula $(\mathrm{mm})$ vs quantitative coronary angiography (QCA) diameter (mm). The CT angiography diameter according to the abbreviated formula ( $\mathrm{mm}$ ) correlated with $\mathrm{QCA}$ diameter $\left(\mathrm{R}^{2}=0.09 \mathrm{p}<0.01\right)$. The mean values were distributed about a straight line given by $\mathrm{y}=0.50 \mathrm{x}+1.86$. of the coronary arterial tree with EBCT. However, it is unrealistic to make an LDP curve or measure the maximum CT value for the short axis at each site of the coronary arteries in routine practice. Therefore, another, easier method was needed. For this, we focused on the maximum CT values for the descending aorta in the corresponding axial images. In theory, it would be better to use the maximum minus baseline $\mathrm{CT}$ values than the maximum $\mathrm{CT}$ values alone. Therefore, we also considered the correlation between the values of $\mathrm{R}^{2}=0.30$ for the average of the 2 sides and 0.37 for the larger of the 2 sides. Those values adjusted the thresholds with the maximum minus baseline CT values in the LDP, which gave less than the maximum CT values alone for the short axes.

To use the maximum CT values for the descending aorta in the corresponding axial images, we applied the abbreviated formula $y=0.5 x-75$ from the straight line given by $y=0.51 x-76.5(y=$ adjusted threshold, $x=$ maximum CT value for the descending aorta in the corresponding axial image). If our abbreviated formula, $y=0.5 x-75$, is to be adopted, it is necessary to measure the maximum CT values for the descending aorta in the corresponding axial images. By including the maximum intensity projection technique, it may be easy to measure the maximum CT values for the descending aorta at each axial image level and acquire information about arterial enhancement for every slice.

The adjusted thresholds correlated with the thresholds obtained from the abbreviated formula, with $\mathrm{R}^{2}=0.33$, and the mean values were distributed about a straight line given by $y=1.02 x-0.4$. The slope of the adjusted thresholds vs the thresholds according to the abbreviated formula was approximately 1.0, and the intercept of this line was only $-0.4 \mathrm{HU}$. In comparing the thresholds according to the abbreviated formula with the adjusted thresholds, the means were similar but the standard deviation was significantly smaller in the thresholds obtained from the abbreviated formula. Therefore, adoption of the thresholds by the abbreviated formula makes it possible to achieve accurate measurements for the evaluation of coronary arteries.

In the scatter plot of the adjusted threshold vs the QCA diameter (Fig 3), the smaller the vessel diameter, the greater the adjusted threshold. Achenbach et al reported that when using $100 \mathrm{HU}$ as the fixed lower threshold, they tended to underestimate small vessel diameters by the partial volume effect ${ }^{15}$ We obtained the opposite result and we speculate that this phenomenon might also be caused by other factors such as vessel type, arterial enhancement, vessel orientation, etc. For example, as shown in Table 3, the mean of the adjusted threshold for the portions of the RCA that were $30 \mathrm{~mm}$ or more from the ostium was $135 \mathrm{HU}$, whereas that of the portions of LCX at $30 \mathrm{~mm}$ or more from the ostium was $94 \mathrm{HU}$. Thus, when we adopted $100 \mathrm{HU}$ as the fixed lower threshold, we overestimated the diameters of the portion of RCA that were $30 \mathrm{~mm}$ or more from the ostium, but underestimated those of the portion of the LCX that were $30 \mathrm{~mm}$ or more from the ostium. The adjusted thresholds correlated very weakly, or non-significantly, to the corresponding diameters measured by QCA, or the distances from the ostium, respectively.

Recently, 16-detector row multislice computed tomography (MSCT) ${ }^{16,17}$ has become available and takes less than $25 \mathrm{~s}$ to image the whole heart, even if a slice thickness less than $1 \mathrm{~mm}$ is selected; this time interval is approximately half to one-third that required for EBCT. Therefore with MSCT, differences in the degree of arterial enhancement would influence the quality of the coronary arterial images to a lesser extent than with EBCT. Some investigators have tried to keep the same degree of arterial enhancement by improving the method of injection of the contrast material, for example via the biphase injection protocol! ${ }^{18}$ If the MSCT coronary angiogram replaced the conventional coronary angiogram only for evaluation of the coronary arterial lumen, the calibration with aortic opacity of the opacified blood would improve the quantitative accuracy of the vessel diameters. Further studies using up-to-date MSCT and EBCT are needed to quantitatively compare the coronary artery diameters with those from QCA.

Although EBCT has worse spatial resolution than MSCT, especially in the through-plane, EBCT delivers a much lower radiation dose than MSCT, so it is still an important diagnostic modality, especially for healthy subjects.

\section{Conclusion}

We measured the adjusted thresholds at which the coronary arterial diameters on EBCT scans were equal to those obtained by a QCA measurement program. We classified a total of 179 sites among 8 patients and 4 vessel types, and further stratified measurements according to positions in relation to the vessel ostium. The means of the adjusted thresholds changed from patient to patient and from vessel 
to vessel.

The adjusted thresholds correlated only very weakly, or non-significantly, with the corresponding QCA measurement diameters, or with the distances from the ostium. However, there was a correlation with maximum CT values for the vessel short axes. Therefore, we decided to use the maximum CT value for the vessel short axis as an index for assessing arterial enhancement. However, it is unrealistic in routine practice to make an LDP curve, or to measure the maximum CT value for the short axis at each site in the coronary artery. Therefore, using the maximum CT values for the descending aorta in the corresponding axial images as a substitute for the maximum CT values for the short axes of coronary arteries, and the abbreviated formula, $y=0.5 x-75$, we developed an accurate and straightforward method for measuring the diameters of coronary arteries independent of arterial enhancement.

\section{Acknowledgments}

This work was supported by the Japan Cardiovascular Research Foundation, Takeda Science Foundation and GE Yokokawa Medical Systems.

\section{References}

1. Moshage WEL, Achenbach S, Seese B, Bachmann K, Kirchgeorg M. Coronary artery stenosis: Three-dimensional imaging with electrocardiographically triggered, contrast agent-enhanced, electron-beam CT. Radiology 1995; 196: 707-714.

2. Achenbach S, Moshage W, Ropers D, Nossen J, Daniel WG. Value of electron-beam computed tomography for the noninvasive detection of high-grade coronary-artery stenoses and occlusions. $N$ Engl J Med 1999; 339: 1964-1971.

3. Budoff MJ, Oudiz RJ, Zalace CP, Bakhsheshi H, Goldberg SL, French WJ, et al. Intravenous three-dimensional coronary angiography using contrast enhanced electron beam computed tomography. Am J Cardiol 1999; 83: 840-845.

4. Shimamoto R, Suzuki J, Nishikawa J, Fujimori Y, Nakamura F, Shin WS, et al. Measuring the diameter of coronary arteries on MR angiograms using spatial profile curves. AJR Am J Roentgenol 1998; 170: $889-893$.

5. Funabashi N, Rubin GD, Kobayashi Y, Shifrin RY, Wexler L, Perlroth M. Accuracy of coronary artery dimensions with electronbeam CT angiography: Comparison of measurement methods (abstract). Radiology 1999; 213(P): 269.

6. Funabashi N, Kobayashi Y, Perlroth M, Rubin GD. Coronary artery; quantitative evaluation of normal artery diameter measurements determined by electron-beam computed tomography in comparison with cine coronary angiography: Initial experience. Radiology 2003; 226: $263-271$.

7. Pomerantsev EV, Kobayashi Y, Fitzgerald PJ, Grube E, Sanders WJ, Alderman EL, et al. Coronary stents: In vitro aspects of an angiographic and ultrasound quantification with in vivo correlation. Circulation 1998; 98: 1495-1503.

8. Gould RG. Principles of ultrafast computed tomography: Historical aspects, mechanism of action, and scanner characteristics. In: Stanford W, Rumberger J, editors. Ultrafast computed tomography in cardiac imaging: Principles and practice. New York: Futura; 1992; $1-15$.

9. Rumberger JA. Ultrafast computed tomography scanning modes, scanning planes and practical aspects of contrast administration. In: Stanford W, Rumberger J, editors. Ultrafast computed tomography in cardiac imaging: Principles and practice. New York: Futura; 1992; $17-24$

10. Chernoff DM, Ritchie CJ, Higgins CB. Evaluation of electron beam CT coronary angiography in healthy subjects. AJR Am J Roentgenol 1997; 169: 93-99.

11. Funabashi N, Misumi K, Ohnishi H, Watanabe M, Suzuki Y, Imai N, et al. Endoluminal perspective volume rendering of coronary arteries using electron-beam computed tomography. Circ J 2003; 67: 1064 1067.

12. Funabashi N, Matsumoto A, Yoshida T, Watanabe S, Misumi K, Masuda Y. Usefulness of three-dimensional visualization of coronary arteries using electron-beam computed tomography data with volume rendering. Jpn Circ J 2000; 64: 644-646.

13. Funabashi N, Kobayashi Y, Rubin GD. Utility of three-dimensional volume rendering images using electron-beam computed tomography to evaluate possible causes of ischemia from an anomalous origin of the right coronary artery from the left sinus of Valsalva. Jpn Circ J 2001; 65: 575-578.

14. Nakanishi T, Kohata M, Miyasaka K, Fukuoka H, Ito K, Imazu M. Virtual endoscopy of coronary arteries using contrast-enhanced ECG-triggered electron beam CT data sets. AJR Am J Roentgenol 2000; 174: $1345-1347$.

15. Achenbach S, Moshage W, Ropers D, Bachmann K. Comparison of vessel diameters in electron beam computed tomography and quantitative coronary angiography. Int J Card Imaging 1998; 14: 1-7.

16. Ropers D, Baum U, Pohle K, Anders K, Ulzheimer S, Ohnesorge B, et al. Detection of coronary artery stenoses with thin-slice multidetector row spiral computed tomography and multiplanar reconstruction. Circulation 2003; 107: 664-666.

17. Sato Y, Kanmatsuse K, Inoue F, Horie T, Kato M, Kusama J, et al. Noninvasive coronary artery imaging by multislice spiral computed tomography. Circ J 2003; 67: 107-111.

18. Fleischmann D, Rubin GD, Bankier AA, Hittmair K. Improved uniformity of aortic enhancement with customized contrast medium injection protocols at CT angiography. Radiology 2000; 214: $363-$ 371. 